Document downloaded from:

http://hdl.handle.net/10251/121417

This paper must be cited as:

Benítez López, J.; Carpitella, S.; Certa, A.; Ilaya-Ayza, AE.; Izquierdo Sebastián, J. (2018). Consistent Clustering of Elements in Large Pairwise Comparison Matrices. Journal of Computational and Applied Mathematics. 343:98-112.

https://doi.org/10.1016/j.cam.2018.04.041

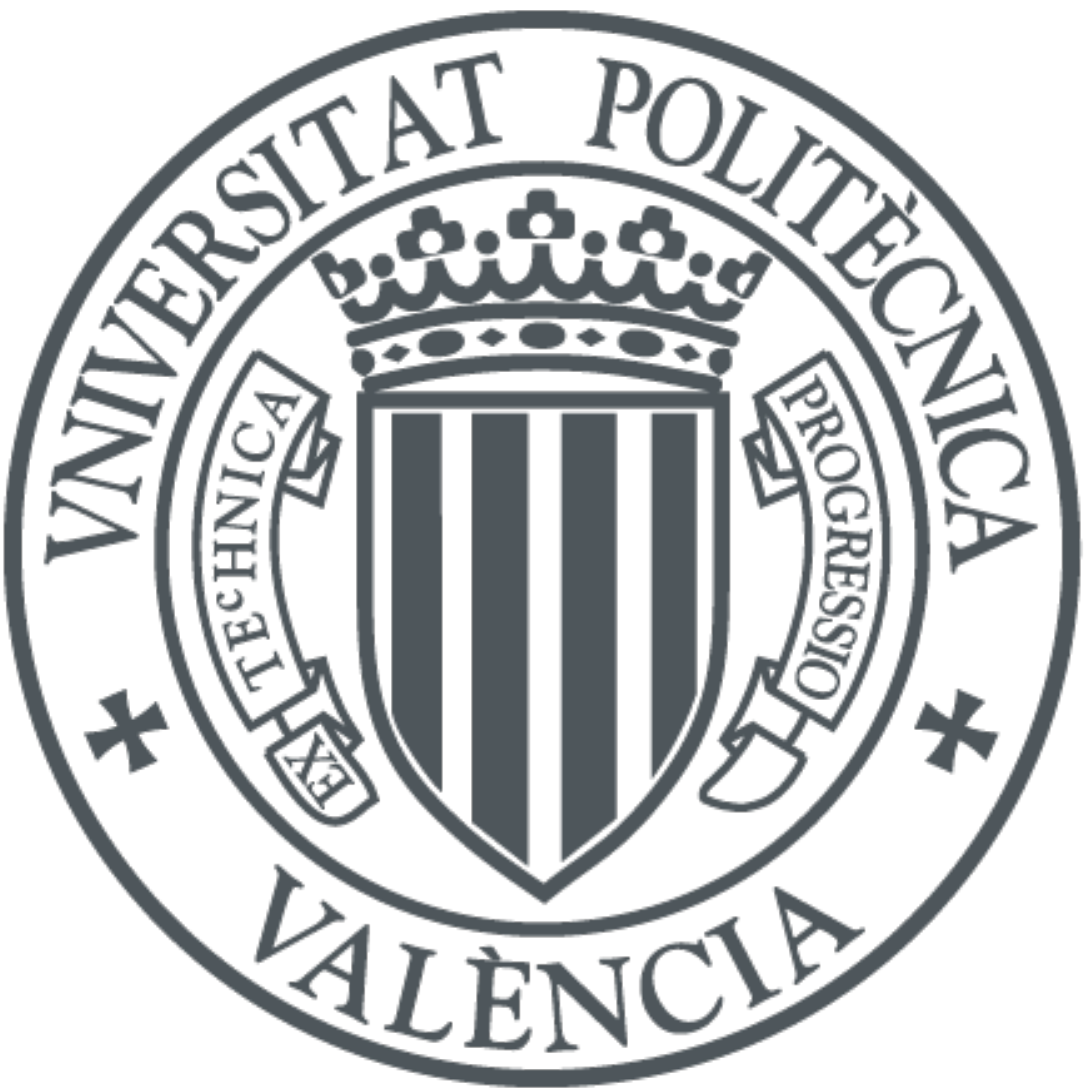

The final publication is available at

http://doi.org/10.1016/j.cam.2018.04.041

Copyright Elsevier

Additional Information 


\title{
Consistent clustering of entries in large pairwise comparison matrices
}

\author{
J. Benítez; S. Carpitella ${ }^{\dagger}$ A. Certa $\stackrel{\text { A.E. Ilaya-Ayza }}{\S}$ J. Izquierdo
}

\begin{abstract}
In multi-attribute decision making the number of decision elements under consideration may be huge, especially for complex, real-world problems. Typically these elements are clustered and then the clusters organized hierarchically to reduce the number of elements to be simultaneously handled. These decomposition methodologies are intended to bring the problem within the cognitive ability of decision makers. However, such methodologies have disadvantages, and it may happen that such a priori clustering is not clear, and/or the problem has previously been addressed without any grouping action. This is the situation for the case study we address, in which a panel of experts gives opinions about the operation of 15 previously established district metered areas in a real water distribution system. Large pairwise comparison matrices may also be found when building comparisons of elements using large bodies of information. In this paper, we address a consistent compression of an AHP comparison matrix that collapses the judgments corresponding to a given number of compared elements. As a result, an a posteriori clustering of various elements becomes possible. In our case study, such a clustering offers several added benefits, including the identification of hidden or unknown criteria to cluster the considered elements of the problem.
\end{abstract}

Keywords: pairwise comparison; AHP; Miller's magic number seven; water distribution system (WDS); management and operation of a WDS; decision-making.

\section{Introduction}

Various decision-making techniques rely on pairwise comparisons (PCs). Traditionally, PCs are provided by experts. Due to human cognitive limitations, and the number of compared elements, the size of pairwise comparison matrices (PCMs) must be small. However, in the current information era, the information collected from databases and the internet is also susceptible to being handled as pairwise comparisons - and these collections can be huge (see $[1,2,3]$, among others). In general, in highly complex problems, the number of elements to be

\footnotetext{
${ }^{*}$ Instituto de Matemática Multidisciplinar, Universitat Politècnica de València, Camino de Vera s/n, 46022 Valencia, Spain. e-mail: jbenitez@mat.upv.es

${ }^{\dagger}$ FluIng, Instituto de Matemática Multidisciplinar, Universidat Politècnica de València Dipartamento dell'Innovazione Industriale e Digitale (DIID), Ingegneria Chimica, Gestionale, Informatica, Meccanica Piazza Marina, 61, 90133 Palermo (PA), Sicilia, Italy

${ }^{\ddagger}$ Dipartamento dell’Innovazione Industriale e Digitale (DIID), Ingegneria Chimica, Gestionale, Informatica, Meccanica Piazza Marina, 61, 90133 Palermo (PA), Sicilia, Italy

${ }_{\S}^{\S}$ Facultad Nacional de Ingeniería, Universidad Técnica de Oruro, Ciudad Universitaria s/n, Oruro, Bolivia

ॠFluIng, Instituto de Matemática Multidisciplinar, Universitat Politècnica de València, Camino de Vera s/n, 46022 Valencia, Spain
} 
compared may be very large. One of the issues limiting PC applicability to large-scale decision problems is the so-called curse of dimensionality, that is, many pairwise comparisons need to be elicited from a decision maker (DM), or built from a body of information. Traditionally, for the analytic hierarchy process (AHP), Saaty [4] recommends that to obtain a reasonable and consistent PCM judgments issued by experts, the number of comparison elements (criteria or alternatives) should be at most seven.

Unfortunately, many decision problems, either coming from expert judgments or from other bodies of information, far exceed this maximum threshold. There are various ways to reduce the size of numerous comparisons. One approach is based on decomposition methodologies $[5,6,7,8,9,10]$. These methodologies overcome the limited cognitive processing power of a DM by decomposing a complex problem into smaller parts to make it more easily understood [11], an aspect we address later on. Unfortunately, these methods also have disadvantages. For example, when a set of elements is decomposed into subsets, the obtained relative weights of the elements are valid only within those subsets. The validity of such weights is not guaranteed at the moment of aggregation. To overcome this problem, a pivot element is arbitrarily selected and assigned to all the subsets and used as a basis for comparing the criteria across all the disjoint subsets. The global weights can then be estimated as in $[5,12,10]$. However, pivot element selection is a challenging issue as the decisions should reduce the number and inconsistency of the pairwise comparisons. These methodologies also lack guidelines for assigning the decision elements for respective subsets since they are made arbitrarily. This does not guarantee that the elements within subsets are independent. This may prevent users from addressing such types of decompositions. Finally, the number of subsets must be known a priori and are subject to DM biases and judgement errors. To overcome these problems, [13] proposes a method to reduce the number and inconsistency of the pairwise comparisons in a large-scale decision set by using a binary integer programming model that segments PC elements into smaller mutually exclusive subsets. The references $[14,15]$ present other examples of clustering alternatives in decision-making problems. However, it may happen that a large comparison matrix may have already been produced that needs further treatment.

Next, in this introduction, we argue that, in the case of expert judgment, PCs with more than seven elements may well make sense and convey valid information, in special cases.

Taking the DM cognitive ability further, in [16] and [17], it is considered that the maximum number of elements handled simultaneously should be seven plus two because of the general limitations on human performance. This limit is widely known in the literature (see Miller [18]) as 'channel capacity', a measure of our ability to process information. This refers to the number of items that can be held in short term memory at any one time.

Miller himself stated that his magic number was for items with one aspect or attribute, and reported this to be true for many tasks. The objective was to determine how well humans can distinguish between differing levels of intensity of particular stimuli, such as frequency, loudness, saltiness, position in an interval, size, and so on. However when more attributes are included, then we can remember more, depending on our familiarity and the complexity of the subject. As expressed in [19], Miller's magical number seven is only true with information that has one memory attribute or function - and we can work with many more. Using one of the examples provided by Miller, Marnell [20] states that 'tonal frequencies according to a provided legend and unravelling the meaning of a map, block...' (this last part of the comparison refers to more complex information structures). Marnell also says that Miller's paper discusses what he calls the span of immediate memory (also known as the capacity of 
our short-term memory), and clarifies that 'the capacity of our short-term memory might well be relevant to our ability to take in and comprehend material at the atomic level ... but at the molecular level ... its relevance is doubtful', and that '... short-term memory is the very stuff of Miller's paper, especially its role in judgment, attention and recall'. Marnell claims that this theory 'needs to be radically updated to bring it into line with current knowledge in cognitive psychology', and he quotes [21] in conclusion that 'a limit of $7 \pm 2$ is yesterday's guesstimate. Today it is $4 \pm 1$ for unrelated items and 15 for... [related concepts]'

We claim that in special cases, for example when a recognized expert, or group of experts, on a subject is confronted with a large number of related items, and there is no clear possibility of clustering them following some homogeneity criteria as suggested in [16], then comparison matrices of more than the traditional $7 \pm 2$ elements may be valid. This is the most salient feature of the case study we address in this paper: a water distribution system (WDS) previously divided into 15 metered areas (sectors) [22] within a project intended to optimally transform intermittent into continuous supply. A team of experts working on a daily basis with the management and operation of the water system were confronted with the problem of clarifying the ease of operation of the individual sectors following their subjective perception about the operating difficulties experienced in each sector, since that level of difficulty would greatly influence the transition process [22]. The comparison matrix was eventually built and, according to our previous arguments, and considering the profound expertise in the problem, the matrix is considered to contain reliable information.

There are various problems associated with dealing with so many elements, for example, when working further with alternatives, as in [23, 24], in various AHP problems or when including the PCs into an analytic network process (ANP) solution of the problem as in [25] and [26], among many others. Moreover, regarding our case study, we have observed certain structured patterns in the matrix. Consequently, we thought of devising a method to consistently shrink or compress the comparison matrix and thus reduce the size of the problem. The paper provides a technique for a posteriori reducing the size of large pairwise comparison matrices, while maintaining consistency. Although originally devised for expert judgment PCMs, the technique can also be used for other large PCMs obtained when handling other pools of data.

The process we describe in this paper is thus a type of pre-process on a large PCM that consistently reduces the number of elements if there is no a priori clear interdependence among the compared elements. It goes without saying that, in the case of judgment-based PCMs, the final approval of the panel of experts is deemed absolutely necessary. In the paper, we develop such a compressing technique so that certain elements may be synthesized to produce a new PCM that:

(i) reduces the size of the problem, thus making it more manageable;

(ii) compresses the PCM while maintaining, in the case of expert-based PCMs, their experience, perception, and consistency; and as a result,

(iii) gathers elements into clusters that may produce additional input to the problem or unveil hidden features.

This size reduction may be useful for a number of goals: for example, to avoid PCs when clarifying alternatives with respect to the sectors; reciprocally, the number of comparisons will also be lower when providing PCs among the sectors for any of the potential alternatives (if the 
ANP methodology is used). For the specific case study we consider later, we additionally point out the potential of this technique for producing interesting results regarding the eventual grouping of sectors that would be useful in the project. Such a method could then be used as an additional criterion in a potentially dynamic sectorization of the network in the process of transforming intermittent into continuous supply.

This PCM compression technique may also be useful in other interesting contexts that consider large PCMs (either from expert judgment or other available information), such as, for example, the merging of companies in shared markets to avoid the whole process of PCs from scratch, once an alliance has been produced.

In the next section we provide a short review of the process of linearization already introduced by the authors [27], and in which our compressing tool is based.

\section{Brief review of the linearization process in AHP}

Throughout this paper, the symbols $\mathscr{M}_{n, m}, \mathscr{M}_{n, m}^{+}, \mathscr{M}_{n}, \mathscr{M}_{n}^{+}$will denote the sets of $n \times m$ real matrices, $n \times m$ positive matrices, $n \times n$ real matrices, and $n \times n$ positive matrices, respectively. Any $k$-dimensional real vector $\mathbf{v} \in \mathbb{R}^{k}$ will be considered a $k \times 1$ matrix (i.e., a column vector). The trace and the transpose of a real matrix $A$ will be denoted by $\operatorname{tr}(A)$ and $A^{T}$, respectively. Finally, the identity matrix of order $n$ will be denoted by $I_{n}$.

Two basic subsets of $\mathscr{M}_{n}^{+}$are particularly interesting in AHP theory, namely, the set of reciprocal matrices and the set of consistent matrices. A matrix $A=\left(a_{i j}\right) \in \mathscr{M}_{n}^{+}$is said to be reciprocal when $a_{i j} a_{j i}=1$ for any $1 \leq i, j \leq n$. A matrix $A=\left(a_{i j}\right) \in \mathscr{M}_{n}^{+}$is said to be consistent when $a_{i j} a_{j k}=a_{i k}$ for any $1 \leq i, j, k \leq n$. It can be easily proven that any consistent matrix is reciprocal.

If $A \in \mathscr{M}_{n}^{+}$is a reciprocal matrix, then there is a unique consistent matrix $X_{A} \in \mathscr{M}_{n}^{+}$ such that

$$
\mathrm{d}\left(A, X_{A}\right) \leq \mathrm{d}(A, X) \quad \forall X \in \mathscr{M}_{n}^{+} \text {consistent. }
$$

See Theorem 2.3 in [27] for the existence of such a matrix and Theorem 2.5 in [27] for an explicit expression of this matrix. In $(1), \mathrm{d}(\cdot, \cdot)$ is the following distance defined in $\mathscr{M}_{n}^{+}$:

$$
\mathrm{d}(M, N)=\|L(M)-L(N)\|_{F},
$$

where $L: \mathscr{M}_{n}^{+} \rightarrow \mathscr{M}_{n},(L(M))_{i j}=\log \left(M_{i j}\right)$ and $\|\cdot\|_{F}$ is the Frobenius norm, i.e., $\|A\|_{F}=$ $\left(\operatorname{tr}\left(A A^{T}\right)\right)^{1 / 2}$ for $A \in \mathscr{M}_{n}$. The inverse of the mapping $L$ will be useful and will be denoted by $E$, which simply is the entrywise exponential (in other parlance, $E: \mathscr{M}_{n} \rightarrow \mathscr{M}_{n}^{+}$is given by $\left.(E(M))_{i j}=\exp \left(M_{i j}\right)\right)$.

Obtaining $X_{A}$ from the reciprocal matrix $A$ is based on a linearization process, described in [27], and summarized in the scheme (2). We will denote by $\mathscr{A}_{n}$ the set of $n \times n$ skew-symmetric matrices and $\mathscr{L}_{n}=\{L(A): A$ is an $n \times n$ consistent matrix $\}$. It can be proven (see Theorem 2.2 in [27]) that $\mathscr{L}_{n}$ is an $(n-1)$-dimensional linear subspace. Evidently, for $A \in \mathscr{M}_{n}^{+}$we have that $A$ is reciprocal if and only if $L(A) \in \mathscr{A}_{n}$. Since any consistent matrix is reciprocal, we obtain $\mathscr{L}_{n} \subset \mathscr{A}_{n}$. Furthermore, we will denote by $p_{n}: \mathscr{M}_{n} \rightarrow \mathscr{M}_{n}$ the orthogonal projection onto $\mathscr{L}_{n}$ when $\mathscr{M}_{n}$ is endowed with the scalar product $\langle A, B\rangle=\operatorname{tr}\left(A B^{T}\right)$.

$$
\underbrace{A}_{\text {Reciprocal }} \stackrel{L}{\longrightarrow} \underbrace{L(A)}_{\in \mathscr{A}_{n}} \stackrel{p_{n}}{\longrightarrow} \underbrace{Y_{A}}_{\in \mathscr{L}_{n}} \stackrel{E}{\longrightarrow} \underbrace{X_{A}=E\left(Y_{A}\right)}_{\text {Consistent }}
$$


From now on, we will denote $\mathbb{1}_{n}$ the column vector of $\mathbb{R}^{n}$ all of whose components are 1 and $U_{n, m}=\mathbb{1}_{n} \mathbb{1}_{m}^{T}$. For the sake of simplicity, $U_{n}=U_{n, n}$.

Although in Theorem 2.5 in [27] an expression of $p_{n}(X)$ was given when $X \in \mathscr{M}_{n}$, as is easily seen in the scheme (2), in AHP theory, it is sufficient to manage $p_{n}(X)$ when $X$ is skew-symmetric. In Theorem 3 in [28] it is proven that if $X \in \mathscr{M}_{n}$ is skew-symmetric, then

$$
p_{n}(X)=\frac{1}{n}\left[\left(X U_{n}\right)-\left(X U_{n}\right)^{T}\right] .
$$

This expression is particularly useful because there is no multiplication to perform.

Another useful mapping is the following: $\phi_{n}: \mathbb{R}^{n} \rightarrow \mathscr{M}_{n}$ given by

$$
\phi_{n}(\mathbf{v})=\mathbf{v} \mathbb{1}_{n}^{T}-\mathbb{1}_{n} \mathbf{v}^{T} .
$$

The following properties of $\phi_{n}$ will be useful (see Theorem 2.2 in [27]): $\phi_{n}$ is linear, $\operatorname{Im} \phi_{n}=$ $\mathcal{L}_{n}$, and $\operatorname{ker} \phi_{n}=\operatorname{span}\left\{\mathbb{1}_{n}\right\}$.

The remainder of the paper is structured as follows. Sections 3 and 4 provide the body of theory upon which the paper is based: including suitable definitions, detailed proofs of lemmas and theorems, as well as synthetic illustrative examples. Section 5 presents the case study, and discusses the obtained results. Finally, Section 6 provides the conclusions.

\section{Clustering of entries in reciprocal matrices}

As said in the introduction, in some concrete situations, it may be a useful approach to collapse several opinions or judgements into just one, while trying to maintain the 'noncollapsed' judgements as faithful to the original as possible. In the following paragraphs we address this problem. Prior to the solution, we present some preliminaries.

If a matrix $A \in \mathscr{M}_{n}^{+}$is reciprocal, to find its closest consistent approximation, we must project $L(A)$ onto $\mathscr{L}_{n}$. As $L(A)$ is skew-symmetric, in the following reasoning, only skewsymmetric matrices are involved instead of reciprocal matrices.

Let $M \in \mathscr{M}_{n+m}$ be a skew-symmetric matrix. Let us partition $M$ as follows

$$
M=\left[\begin{array}{cc}
M_{1} & -M_{2} \\
M_{2}^{T} & M_{3}
\end{array}\right] \in \mathscr{M}_{n+m}, \quad M_{1} \in \mathscr{M}_{n}, M_{3} \in \mathscr{M}_{m}
$$

$M_{1}$ and $M_{3}$ being skew-symmetric (this is because $M$ is skew-symmetric). The relations between the last $m$ judgments of $M$ are reflected in the block $M_{3}$, and the relations between the first $n$ judgments and the last $m$ judgments are reflected in the block $M_{2}$ (let us note that $M_{2}$ can be a non-square matrix -in case $n \neq m$ holds).

If we want to collapse the $i$-th judgments $(i=n+1, \ldots, n+m)$ of $M$ to only one, then we are forced to consider the following $n+1$ square block matrix:

$$
N=\left[\begin{array}{cc}
M_{1} & -\mathbf{v} \\
\mathbf{v}^{T} & 0
\end{array}\right] \in \mathscr{M}_{n+1}, \quad \mathbf{v} \in \mathbb{R}^{n}
$$

Observe that the 'north-west' blocks of $M$ and $N$ must be equal if we want that the preservation of the judgments in this collapsed matrix $N$ to be as faithful possible. Our purpose is: how to find vector $\mathbf{v}$ ? 
If this 'collapse' is coherent, then the information concerning the $1, \ldots, n$ judgments must not be changed. In other words, since the orthogonal projection onto $\mathscr{L}_{n+m}$ and $\mathscr{L}_{n+1}$ provide the best approximations, if

$$
p_{n+m}(M)=\left[\begin{array}{cc}
X & -Y \\
Y^{T} & Z
\end{array}\right], \quad p_{n+1}(N)=\left[\begin{array}{cc}
X^{\prime} & -\mathbf{y} \\
\mathbf{y}^{T} & 0
\end{array}\right], \quad X, X^{\prime} \in \mathscr{M}_{n},
$$

then one must have $X=X^{\prime}$.

Once the vector $\mathbf{v}$ in matrix $N$ written in (6) is found, the expert(s) that filled the matrix $M$ must be asked (in a feedback process) if this vector $\mathbf{v}$ (which reflects the relations between the $1, \ldots, n$ judgements and the collapsed one) is adequate.

The next auxiliary lemma will be useful to prove the main results of this paper.

Lemma 1. If $M \in \mathscr{M}_{n}$ is skew-symmetric, then $U_{m, n} M \mathbb{1}_{n}=\mathbf{0}$.

Proof. Let $\left\{\mathbf{e}_{1}, \ldots, \mathbf{e}_{m}\right\}$ be the standard basis of $\mathbb{R}^{m}$. The lemma will be proven if we demonstrate $\mathbf{e}_{i}^{T} U_{m, n} M \mathbb{1}_{n}=0$ for $i=1, \ldots, m$. Since $\mathbf{e}_{i}^{T} U_{m, n}=\mathbb{1}_{n}^{T}$ we obtain $\mathbf{e}_{i}^{T} U_{m, n} M \mathbb{1}_{n}=$ $\mathbb{1}_{n}^{T} M \mathbb{1}_{n}$. Let us bear in mind that $\mathbb{1}_{n}^{T} M \mathbb{1}_{n}$ is a scalar, and so, coincides with its transpose. Since $M=-M^{T}$ we have $\mathbb{1}_{n}^{T} M \mathbb{1}_{n}=\left(\mathbb{1}_{n}^{T} M \mathbb{1}_{n}\right)^{T}=\mathbb{1}_{n}^{T} M^{T} \mathbb{1}_{n}=-\mathbb{1}_{n}^{T} M \mathbb{1}_{n}$. Hence $\mathbb{1}_{n}^{T} M \mathbb{1}_{n}=0$ and the proof of the lemma is ended.

Also, the next result (Theorem 4, [28]) will play an essential role in the sequel, and we include it for the sake of readability. Let us recall that the mapping $\phi_{n}$ is defined in (4) and $p_{n}: \mathscr{M}_{n} \rightarrow \mathscr{L}_{n}$ denotes the orthogonal projection onto $\mathscr{L}_{n}$.

Theorem 1. Let $M \in \mathscr{M}_{n}$ be skew-Hermitian and $\mathbf{v} \in \mathbb{R}^{n}$. Then $\phi_{n}(\mathbf{v})=p_{n}(M)$ if and only if there exists $\alpha \in \mathbb{R}$ such that $\mathbf{v}=\frac{1}{n} M \mathbb{1}_{n}+\alpha \mathbb{1}_{n}$.

The main results of the paper follow below.

Theorem 2. Let $M \in \mathscr{M}_{n+m}$ and $N \in \mathscr{M}_{n+1}$ be skew-symmetric matrices decomposed as in (5) and (6), respectively. Let $p_{n+m}(M)$ and $p_{n+1}(N)$ be decomposed as in (7). Then the following conditions are equivalent:

(i) $X=X^{\prime}$

(ii) There exists $\alpha \in \mathbb{R}$ such that

$$
\mathbf{v}=\frac{n+1}{n+m} M_{2} \mathbb{1}_{m}+\frac{m-1}{n+m} M_{1} \mathbb{1}_{n}+\alpha \mathbb{1}_{n}
$$

Under this equivalence, one has

$$
\mathbf{y}=\frac{1}{n+m}\left[-M_{1} \mathbb{1}_{n}+\left(I_{n}+U_{n}\right) M_{2} \mathbb{1}_{m}\right]+\alpha \mathbb{1}_{n} .
$$

Proof. Let us recall that

$$
p_{n+m}(M)=\frac{1}{n+m}\left[\left(M U_{n+m}\right)-\left(M U_{n+m}\right)^{T}\right] .
$$


Since

$$
M U_{n+m}=\left[\begin{array}{cc}
M_{1} & -M_{2} \\
M_{2}^{T} & M_{3}
\end{array}\right]\left[\begin{array}{cc}
U_{n} & U_{n, m} \\
U_{m, n} & U_{m}
\end{array}\right]=\left[\begin{array}{cc}
M_{1} U_{n}-M_{2} U_{m, n} & M_{1} U_{n, m}-M_{2} U_{m} \\
M_{2}^{T} U_{n}+M_{3} U_{m, n} & M_{2}^{T} U_{n, m}+M_{3} U_{m}
\end{array}\right] .
$$

Therefore, from (7) and (9) we obtain

$$
X=\frac{1}{n+m}\left[\left(M_{1} U_{n}-M_{2} U_{m, n}\right)-\left(M_{1} U_{n}-M_{2} U_{m, n}\right)^{T}\right] .
$$

We have, from the definition of $\phi_{n}$ given in (4),

$$
\begin{aligned}
M_{2} U_{m, n}-\left(M_{2} U_{m, n}\right)^{T} & =M_{2}\left(\mathbb{1}_{m} \mathbb{1}_{n}^{T}\right)-\left[M_{2}\left(\mathbb{1}_{m} \mathbb{1}_{n}^{T}\right)\right]^{T} \\
& =\left(M_{2} \mathbb{1}_{m}\right) \mathbb{1}_{n}^{T}-\left[\left(M_{2} \mathbb{1}_{m}\right) \mathbb{1}_{n}^{T}\right]^{T} \\
& =\left(M_{2} \mathbb{1}_{m}\right) \mathbb{1}_{n}^{T}-\mathbb{1}_{n}\left(M_{2} \mathbb{1}_{m}\right)^{T} \\
& =\phi_{n}\left(M_{2} \mathbb{1}_{m}\right) .
\end{aligned}
$$

Hence,

$$
X=\frac{1}{n+m}\left[\left(M_{1} U_{n}\right)-\left(M_{1} U_{n}\right)^{T}-\phi_{n}\left(M_{2} \mathbb{1}_{m}\right)\right]=\frac{1}{n+m}\left[n p_{n}\left(M_{1}\right)-\phi_{n}\left(M_{2} \mathbb{1}_{m}\right)\right] .
$$

Analogously we obtain

$$
X^{\prime}=\frac{1}{n+1}\left[\left(M_{1} U_{n}-\mathbf{v} \mathbb{1}_{n}^{T}\right)-\left(M_{1} U_{n}-\mathbf{v} \mathbb{1}_{n}^{T}\right)^{T}\right]=\frac{1}{n+1}\left[n p_{n}\left(M_{1}\right)-\phi_{n}(\mathbf{v})\right] .
$$

(i) $\Rightarrow$ (ii). From $X=X^{\prime},(11)$, and (12) we obtain

$$
\frac{1}{n+m}\left[n p_{n}\left(M_{1}\right)-\phi_{n}\left(M_{2} \mathbb{1}_{m}\right)\right]=\frac{1}{n+1}\left[n p_{n}\left(M_{1}\right)-\phi_{n}(\mathbf{v})\right]
$$

Therefore, by recalling that $\phi_{n}$ and $p_{n}$ are linear mappings

$$
\phi_{n}\left(\frac{1}{n+1} \mathbf{v}-\frac{1}{n+m} M_{2} \mathbb{1}_{m}\right)=p_{n}\left(\left(\frac{n}{n+1}-\frac{n}{n+m}\right) M_{1}\right) .
$$

Let us recall that $M_{1}$ is skew-symmetric. From Theorem 1 , there exists $\alpha \in \mathbb{R}$, such that

$$
\frac{1}{n+1} \mathbf{v}-\frac{1}{n+m} M_{2} \mathbb{1}_{m}=\frac{1}{n}\left(\frac{n}{n+1}-\frac{n}{n+m}\right) M_{1} \mathbb{1}_{n}+\alpha \mathbb{1}_{n} .
$$

By performing some easy computations and renaming $(n+1) \alpha$ to $\alpha$, we obtain the expression of $\mathbf{v}$ given in the statement of the theorem.

(ii) $\Rightarrow$ (i): We know that exists $\alpha \in \mathbb{R}$, such that

$$
\mathbf{v}-\frac{n+1}{n+m} M_{2} \mathbb{1}_{m}=\frac{1}{n} \frac{n(m-1)}{n+m} M_{1} \mathbb{1}_{n}+\alpha \mathbb{1}_{n} .
$$

By Theorem 1 and since $\frac{n(m-1)}{n+m} M_{1}$ is skew-symmetric, we obtain

$$
\phi_{n}\left(\mathbf{v}-\frac{n+1}{n+m} M_{2} \mathbb{1}_{m}\right)=p_{n}\left(\frac{n(m-1)}{n+m} M_{1}\right) .
$$


Now, the linearity of $p_{n}$ and $\phi_{n}$, together with (11) and (12) lead to

$$
\begin{aligned}
X^{\prime} & =\frac{1}{n+1}\left[n p_{n}\left(M_{1}\right)-\phi_{n}(\mathbf{v})\right] \\
& =\frac{1}{n+1}\left[n p_{n}\left(M_{1}\right)-\left(\phi_{n}\left(\frac{n+1}{n+m} M_{2} \mathbb{1}_{m}\right)+p_{n}\left(\frac{n(m-1)}{n+m} M_{1}\right)\right)\right] \\
& =\frac{n}{n+m} p_{n}\left(M_{1}\right)-\frac{1}{n+m} \phi_{n}\left(M_{2} \mathbb{1}_{m}\right) \\
& =X
\end{aligned}
$$

This proves the first part of the theorem.

To find $\mathbf{y}$, which appears in $(7)$, we use $p_{n+1}(N)=\left[N U_{n+1}-\left(N U_{n+1}\right)^{T}\right] /(n+1)$. Since (we have marked with $\star$ the entries that we are not interested in)

$$
N U_{n+1}=\left[\begin{array}{cc}
M_{1} & -\mathbf{v} \\
\mathbf{v}^{T} & 0
\end{array}\right]\left[\begin{array}{cc}
U_{n} & \mathbb{1}_{n} \\
\mathbb{1}_{n}^{T} & 1
\end{array}\right]=\left[\begin{array}{cc}
\star & M_{1} \mathbb{1}_{n}-\mathbf{v} \\
\mathbf{v}^{T} U_{n} & \star
\end{array}\right]
$$

we obtain (recall that $U_{n}$ is symmetric)

$$
p_{n+1}(N)=\frac{1}{n+1}\left[\begin{array}{cc}
\star & M_{1} \mathbb{1}_{n}-\mathbf{v}-U_{n} \mathbf{v} \\
\star & \star
\end{array}\right] .
$$

Hence, by using $U_{n} \mathbb{1}_{n}=n \mathbb{1}_{n}$, the first part of the theorem, (7), and Lemma 1 ,

$$
\begin{aligned}
(n+1) \mathbf{y}=- & M_{1} \mathbb{1}_{n}+\mathbf{v}+U_{n} \mathbf{v} \\
=- & M_{1} \mathbb{1}_{n}+\frac{n+1}{n+m} M_{2} \mathbb{1}_{m}+\frac{m-1}{n+m} M_{1} \mathbb{1}_{n}+\alpha \mathbb{1}_{n}+ \\
& +U_{n}\left(\frac{n+1}{n+m} M_{2} \mathbb{1}_{m}+\frac{m-1}{n+m} M_{1} \mathbb{1}_{n}+\alpha \mathbb{1}_{n}\right) \\
=- & \frac{n+1}{n+m} M_{1} \mathbb{1}_{n}+\frac{n+1}{n+m} M_{2} \mathbb{1}_{m}+(n+1) \alpha \mathbb{1}_{n}+\frac{n+1}{n+m} U_{n} M_{2} \mathbb{1}_{m} .
\end{aligned}
$$

The proof is finished.

It is noteworthy that the vectors $\mathbf{v}$ and $\mathbf{y}$ in Theorem 2 are independent in block $M_{3}$. In other words, to collapse the criteria $n+1, \ldots, n+m$ we can ignore the pairwise comparisons among these criteria.

However, the arbitrariness of the scalar $\alpha$ appearing in Theorem 2 leads us to impose another condition. Let us motivate it with the following example. Let $M_{1} \in \mathcal{M}_{n}$ be skewsymmetric and $\mathbf{v} \in \mathbb{R}^{n}$. Set $M=N=\left[\begin{array}{cc}M_{1} & -\mathbf{v} \\ \mathbf{v}^{T} & 0\end{array}\right]$. By using Theorem 2 (observe that $m=1$ ) we obtain that the presence of $\alpha$ is awkward. If we look at (7), we can think on $\mathbf{y}$ as a 'mixture' of $Y$. In fact, we shall impose that $\mathbf{y}$ is the arithmetic mean of $Y$.

Theorem 3. Let $M \in \mathscr{M}_{n+m}$ and $N \in \mathscr{M}_{n+1}$ be skew-symmetric matrices decomposed as in (5) and (6), respectively. Let $p_{n+m}(M)$ and $p_{n+1}(N)$ be decomposed as in (7) with $X=X^{\prime}$. If

$$
\frac{1}{m} Y \mathbb{1}_{m}=\mathbf{y},
$$


then the scalar $\alpha$ appearing in Theorem 2 is

$$
\alpha=S \frac{1-m}{(n+m) m}
$$

where $S$ is the sum of all the components of $M_{2}$.

Proof. We shall use the notation of Theorem 2 and its proof. By (9) and (10),

$$
\begin{aligned}
-Y \mathbb{1}_{m} & =\left[\text { Block }(1,2) \text { of } p_{n+m}(M)\right] \mathbb{1}_{m} \\
& =\frac{1}{n+m}\left[M_{1} U_{n, m}-M_{2} U_{m}-\left(M_{2}^{T} U_{n}+M_{3} U_{m, n}\right)^{T}\right] \mathbb{1}_{m} \\
& =\frac{1}{n+m}\left[M_{1} U_{n, m} \mathbb{1}_{m}-M_{2} U_{m} \mathbb{1}_{m}-U_{n} M_{2} \mathbb{1}_{m}-U_{n, m} M_{3}^{T} \mathbb{1}_{m}\right] .
\end{aligned}
$$

Let us observe that $U_{n, m} \mathbb{1}_{m}=m \mathbb{1}_{n}, U_{m} \mathbb{1}_{m}=m \mathbb{1}_{m}$ and $U_{n, m} M_{3}^{T} \mathbb{1}_{m}=0$ (because $M_{3}$ is skew-symmetric and using Lemma 1). Therefore,

$$
\begin{aligned}
-\frac{1}{m} Y \mathbb{1}_{m}= & \frac{1}{n+m}\left[M_{1} \mathbb{1}_{n}-M_{2} \mathbb{1}_{m}-\frac{1}{m} U_{n} M_{2} \mathbb{1}_{m}\right] \\
= & \frac{1}{n+m}\left[M_{1} \mathbb{1}_{n}-M_{2} \mathbb{1}_{m}-U_{n} M_{2} \mathbb{1}_{m}\right]+\frac{1}{n+m} U_{n} M_{2} \mathbb{1}_{m} \\
& -\alpha \mathbb{1}_{n}+\alpha \mathbb{1}_{n}-\frac{1}{m(n+m)} U_{n} M_{2} \mathbb{1}_{m} \\
= & -\mathbf{y}+\frac{1}{n+m}\left(1-\frac{1}{m}\right) U_{n} M_{2} \mathbb{1}_{m}+\alpha \mathbb{1}_{n} .
\end{aligned}
$$

But, as one can easily see, $U_{n} M_{2} \mathbb{1}_{m}=S \mathbb{1}_{n}$, where $S$ is the sum of all of components of $M_{2}$. Therefore,

$$
-\frac{1}{m} Y \mathbb{1}_{m}=-\mathbf{y}+\left(\frac{m-1}{m(n+m)} S+\alpha\right) \mathbb{1}_{n}
$$

The proof is finished.

Definition 1. Let $M \in \mathscr{M}_{n+m}$ be a skew-symmetric matrix decomposed as in (5). If $\alpha \in \mathbb{R}$ is given by (13), $\mathbf{v} \in \mathbb{R}^{n}$ is given by (8), and $N \in \mathscr{M}_{n+1}$ is given by (6), then we say that the collapse of the last $m$ judgements of $M$ produces $N$.

Example 1. We close this section with a rather artificial example. Let $A$ be the following reciprocal matrix

$$
A=\left[\begin{array}{cc|cc}
1 & 3 & 2 & 4 \\
1 / 3 & 1 & 2 & 3 \\
\hline 1 / 2 & 1 / 2 & 1 & 2 \\
1 / 4 & 1 / 3 & 1 / 2 & 1
\end{array}\right]
$$

If we want to collapse the third and fourth judgements, we use Theorems 2 and 3 for $n=$ $m=2$.

$$
M=L(A)=\left[\begin{array}{cc|cc}
0 & 1.0986 & 0.6932 & 1.3863 \\
-1.0986 & 0 & 0.6932 & 1.0986 \\
\hline-0.6932 & -0.6932 & 0 & 0.6932 \\
-1.3863 & -1.0986 & -0.6932 & 0
\end{array}\right]=\left[\begin{array}{cc}
M_{1} & -M_{2} \\
M_{2}^{T} & M_{3}
\end{array}\right]
$$


By Theorem 3, we get $\alpha=0.4839$. By Theorem 2, we get

$$
\mathbf{v}=\left[\begin{array}{ll}
-1.3774-1.1617
\end{array}\right]^{T} \text {. }
$$

Therefore, the collapsed matrix $N$ given by Definition 1 is

$$
N=\left[\begin{array}{cc}
M_{1} & -\mathbf{v} \\
\mathbf{v}^{T} & 0
\end{array}\right]=\left[\begin{array}{ccc}
0 & 1.0986 & 1.3774 \\
-1.0986 & 0 & 1.1617 \\
-1.3774 & -1.1617 & 0
\end{array}\right]
$$

Coming back to the comparison matrices, we obtain that the collapse of the 3rd and 4th judgements produces

$$
E(N)=\left[\begin{array}{ccc}
1 & 3 & 3.9647 \\
1 / 3 & 1 & 3.1952 \\
0.25223 & 0.31297 & 1
\end{array}\right]
$$

Now is the time for the expert to decide if he/she agrees with this new comparison matrix.

\section{Clustering of entries in consistent matrices}

Let us recall that if $A=\left[a_{i j}\right] \in \mathscr{M}_{n}$ is a consistent matrix, then exists $\mathbf{v}=\left[v_{1} \cdots v_{n}\right]^{T} \in \mathbb{R}^{n}$ such that $a_{i j}=v_{i} v_{j}^{-1}$ for all $1 \leq i, j \leq n$. This vector $\mathbf{v}$ is the priority vector of the matrix $A$, and it is easily checked that $\mathbf{v}$ is an eigenvector of $A$ associated to the eigenvalue $n$. This eigenvalue $n$ is the Perron eigenvalue of the positive matrix $A$ (see [29] for the complete details).

Before studying how to collapse several judgments in a consistent matrix, let us see a general useful fact: Let $A \in \mathscr{M}_{n}$ be a consistent matrix. If $\mathbf{z} \in \mathbb{R}^{n}$ is the priority vector of $A$, then $L(A)=\phi_{n}(L(\mathbf{z}))$. In fact: since $a_{i j}=z_{i} z_{j}^{-1}$ we have $\log \left(a_{i j}\right)=\log \left(z_{i}\right)-\log \left(z_{j}\right)$ for all $1 \leq i, j \leq n$, and therefore, $L(A)=\phi_{n}(L(\mathbf{z}))$.

Let $A \in \mathscr{M}_{n+m}^{+}$be a consistent matrix and let us partition $A$ as follows:

$$
A=\left[\begin{array}{cc}
A_{1} & A_{2} \\
A_{3} & A_{4}
\end{array}\right], \quad A_{1} \in \mathscr{M}_{n}, A_{4} \in \mathscr{M}_{m}
$$

It is evident that $A_{1}$ is consistent (it is the comparison matrix of the $1, \ldots, n$ judgements). Also, $A_{4}$ is the comparison matrix of the $n+1, \ldots, n+m$ judgements, which is also consistent. Let $\mathbf{z} \in \mathbb{R}^{n+m}$ be the priority vector of $A$. Let us decompose $\mathbf{z}=\left[\begin{array}{l}\mathbf{z}_{1} \\ \mathbf{z}_{2}\end{array}\right]$, where $\mathbf{z}_{1} \in \mathbb{R}^{n}$. Now, one has $L(A)=\phi_{n+m}(L(\mathbf{z}))$. Hence

$$
\begin{aligned}
L(A) & =\left[\begin{array}{ll}
L\left(A_{1}\right) & L\left(A_{2}\right) \\
L\left(A_{3}\right) & L\left(A_{4}\right)
\end{array}\right]=\phi_{n+m}(L(\mathbf{z}))=L(\mathbf{z}) \mathbb{1}_{n+m}^{T}-\mathbb{1}_{n+m} L(\mathbf{z})^{T} \\
& =\left[\begin{array}{l}
L\left(\mathbf{z}_{1}\right) \\
L\left(\mathbf{z}_{2}\right)
\end{array}\right]\left[\begin{array}{ll}
\mathbb{1}_{n}^{T} & \mathbb{1}_{m}^{T}
\end{array}\right]-\left[\begin{array}{c}
\mathbb{1}_{n} \\
\mathbb{1}_{m}
\end{array}\right]\left[\begin{array}{ll}
L\left(\mathbf{z}_{1}\right)^{T} & L\left(\mathbf{z}_{2}\right)^{T}
\end{array}\right] \\
& =\left[\begin{array}{cc}
L\left(\mathbf{z}_{1}\right) \mathbb{1}_{n}^{T}-\mathbb{1}_{n} L\left(\mathbf{z}_{1}\right)^{T} & L\left(\mathbf{z}_{1}\right) \mathbb{1}_{m}^{T}-\mathbb{1}_{n} L\left(\mathbf{z}_{2}\right)^{T} \\
L\left(\mathbf{z}_{2}\right) \mathbb{1}_{n}^{T}-\mathbb{1}_{m} L\left(\mathbf{z}_{1}\right)^{T} & L\left(\mathbf{z}_{2}\right) \mathbb{1}_{m}^{T}-\mathbb{1}_{m} L\left(\mathbf{z}_{2}\right)^{T}
\end{array}\right] \\
& =\left[\begin{array}{cc}
\phi_{n}\left(L\left(\mathbf{z}_{1}\right)\right) & L\left(\mathbf{z}_{1}\right) \mathbb{1}_{m}^{T}-\mathbb{1}_{n} L\left(\mathbf{z}_{2}\right)^{T} \\
L\left(\mathbf{z}_{2}\right) \mathbb{1}_{n}^{T}-\mathbb{1}_{m} L\left(\mathbf{z}_{1}\right)^{T} & \phi_{m}\left(L\left(\mathbf{z}_{2}\right)\right)
\end{array}\right] .
\end{aligned}
$$


Therefore, $\mathbf{z}_{1}$ is the priority vector of $A_{1}$ and $\mathbf{z}_{2}$ is the priority vector of $A_{4}$.

Theorem 4. Let $A \in \mathscr{M}_{n+m}$ be a consistent matrix decomposed as in (14) whose priority vector is $\left[\begin{array}{llll}z_{1} & z_{2} & \cdots & z_{n+m}\end{array}\right]^{T}$ and $M=L(A)$ be decomposed as in (5). Let $N$ be produced by the collapse of the last $m$ judgements of $M$, and finally, let us denote $\mathbf{w}_{1}=\left[\begin{array}{llll}\log z_{1} & \cdots & \log z_{n}\end{array}\right]^{T}$ and $s_{2}=\log z_{n+1}+\cdots+\log z_{n+m}$. Then

(i) $N=\left[\begin{array}{cc}M_{1} & -\mathbf{v} \\ \mathbf{v}^{T} & 0\end{array}\right]$, where $\mathbf{v}=-\mathbf{w}_{1}+\frac{s_{2}}{m} \mathbb{1}_{n}$.

(ii) $p_{n+1}(N)=N$. We obtain that $N \in \mathscr{L}_{n+1}$, or equivalently, $E(N)$ is a consistent matrix.

(iii) The priority vector for $E(N)$ is $\left[\begin{array}{llll}z_{1} & \cdots & z_{n} & \sqrt[m]{z_{n+1}} \cdots z_{n+m}\end{array}\right]^{T}$.

Proof. Let $\mathbf{z}$ be the priority vector of $A$ decomposed as in the previous paragraph. We shall denote $\mathbf{w}_{1}=L\left(\mathbf{z}_{1}\right)$ and $\mathbf{w}_{2}=L\left(\mathbf{z}_{2}\right)$. We also denote $s_{1}=\mathbf{w}_{1}^{T} \mathbb{1}_{n}$ and $s_{2}=\mathbf{w}_{2}^{T} \mathbb{1}_{m}$ (observe that $s_{i}$ is the sum of the components of $\mathbf{w}_{i}$ for $\left.i=1,2\right)$.

We shall apply Theorem 3 and Theorem 2 to obtain vector v. From decompositions (5) and (15) we obtain

$$
M_{1}=\mathbf{w}_{1} \mathbb{1}_{n}^{T}-\mathbb{1}_{n} \mathbf{w}_{1}^{T} \quad \text { and } \quad-M_{2}=\mathbf{w}_{1} \mathbb{1}_{m}^{T}-\mathbb{1}_{n} \mathbf{w}_{2}^{T} .
$$

Now, observe that $\mathbb{1}_{n}^{T} \mathbb{1}_{n}=n$ and $\mathbf{w}_{1}^{T} \mathbb{1}_{n}$ is an scalar (which commutes with any matrix). So,

$$
M_{1} \mathbb{1}_{n}=\left(\mathbf{w}_{1} \mathbb{1}_{n}^{T}-\mathbb{1}_{n} \mathbf{w}_{1}^{T}\right) \mathbb{1}_{n}=\mathbf{w}_{1} \mathbb{1}_{n}^{T} \mathbb{1}_{n}-\mathbb{1}_{n} \mathbf{w}_{1}^{T} \mathbb{1}_{n}=n \mathbf{w}_{1}-s_{1} \mathbb{1}_{n}
$$

and, analogously,

$$
-M_{2} \mathbb{1}_{m}=\left(\mathbf{w}_{1} \mathbb{1}_{m}^{T}-\mathbb{1}_{n} \mathbf{w}_{2}^{T}\right) \mathbb{1}_{m}=\mathbf{w}_{1} \mathbb{1}_{m}^{T} \mathbb{1}_{m}-\mathbb{1}_{n} \mathbf{w}_{2}^{T} \mathbb{1}_{m}=m \mathbf{w}_{1}-s_{2} \mathbb{1}_{n} .
$$

Firstly, we obtain the value of $\alpha$ in Theorem 3. It is easy to see that the sum of the entries of $M_{2}$ is

$$
S=\mathbb{1}_{n}^{T} M_{2} \mathbb{1}_{m}=\mathbb{1}_{n}^{T}\left(-m \mathbf{w}_{1}+s_{2} \mathbb{1}_{n}\right)=-m s_{1}+n s_{2}
$$

Therefore,

$$
\alpha=\left(m s_{1}-n s_{2}\right) \frac{m-1}{m(n+m)} .
$$

Now, we find an expression for vector $\mathbf{v}$ appearing in Theorem 2 :

$$
\begin{aligned}
\mathbf{v} & =\frac{n+1}{n+m} M_{2} \mathbb{1}_{m}+\frac{m-1}{n+m} M_{1} \mathbb{1}_{n}+\alpha \mathbb{1}_{n} \\
& =\frac{n+1}{n+m}\left(-m \mathbf{w}_{1}+s_{2} \mathbb{1}_{n}\right)+\frac{m-1}{n+m}\left(n \mathbf{w}_{1}-s_{1} \mathbb{1}_{n}\right)+\left(m s_{1}-n s_{2}\right) \frac{m-1}{m(n+m)} \mathbb{1}_{n} \\
& =-\mathbf{w}_{1}+\frac{1}{n+m}\left[s_{2}(n+1)-s_{1}(m-1)+\left(m s_{1}-n s_{2}\right) \frac{m-1}{m}\right] \mathbb{1}_{n} \\
& =-\mathbf{w}_{1}+\frac{s_{2}}{m} \mathbb{1}_{n} .
\end{aligned}
$$

Item (i) is proven. To prove item (ii), we seek for a simplified expression of the vector $\mathbf{y}$ appearing in Theorem 2. Before doing this, we simplify $U_{n} M_{2} \mathbb{1}_{m}$ :

$$
\begin{aligned}
-U_{n} M_{2} \mathbb{1}_{m} & =U_{n}\left(m \mathbf{w}_{1}-s_{2} \mathbb{1}_{n}\right) \\
& =m \mathbb{1}_{n} \mathbb{1}_{n}^{T} \mathbf{w}_{1}-s_{2} \mathbb{1}_{n} \mathbb{1}_{n}^{T} \mathbb{1}_{n}=m \mathbb{1}_{n} s_{1}-s_{2} \mathbb{1}_{n} n=\left(m s_{1}-n s_{2}\right) \mathbb{1}_{n} .
\end{aligned}
$$


Now,

$$
\begin{aligned}
-\mathbf{y} & =\frac{1}{n+m}\left[M_{1} \mathbb{1}_{n}-\left(I_{n}+U_{n}\right) M_{2} \mathbb{1}_{m}\right]-\alpha \mathbb{1}_{n} \\
& =\frac{1}{n+m}\left[n \mathbf{w}_{1}-s_{1} \mathbb{1}_{n}+m \mathbf{w}_{1}-s_{2} \mathbb{1}_{n}+\left(m s_{1}-n s_{2}\right) \mathbb{1}_{n}\right]-\alpha \mathbb{1}_{n} \\
& =\mathbf{w}_{1}+\left(\frac{m s_{1}-n s_{2}}{n+m}-\frac{s_{1}+s_{2}}{n+m}-\alpha\right) \mathbb{1}_{n} \\
& =\mathbf{w}_{1}+\left(\frac{m s_{1}-n s_{2}}{n+m}-\frac{s_{1}+s_{2}}{n+m}-\left(m s_{1}-n s_{2}\right) \frac{m-1}{m(n+m)}\right) \mathbb{1}_{n} \\
& =\mathbf{w}_{1}+\frac{m s_{1}-n s_{2}-m\left(s_{1}+s_{2}\right)}{m(n+m)} \mathbb{1}_{n} \\
& =\mathbf{w}_{1}-\frac{s_{2}}{m} \mathbb{1}_{n} .
\end{aligned}
$$

Observe that since $A$ is consistent, we have $M=L(A) \in \mathscr{L}_{n+m}$. Therefore, $p_{n+m}(M)=M$. From (5) and (7) we have $M_{1}=X$. From Theorem 2 we have $X=X^{\prime}$. From the above computations we obtain $\mathbf{v}=\mathbf{y}$. Hence, the expressions for $N$ and $p_{n+1}(N)$ given in $(6)$ and (7) prove item (ii).

To prove item (iii), we recall that the priority vector of a consistent matrix is just a scalar multiple of any of its columns, and therefore, the priority vector for $E(N)$ can be regarded as a multiple of its last column, which is

$$
\left[\begin{array}{c}
E(-\mathbf{v}) \\
1
\end{array}\right]=\left[\begin{array}{c}
E\left(\mathbf{w}_{1}-\frac{s_{2}}{m} \mathbb{1}_{n}\right) \\
1
\end{array}\right]
$$

Observe that the $i$ th component of $\mathbf{w}_{1}-\frac{s_{2}}{m} \mathbb{1}_{m}$ is given by

$$
\log z_{i}-\frac{\log z_{n+1}+\cdots+\log z_{n+m}}{m}=\log \left(\frac{z_{i}}{\sqrt[m]{z_{n+1} \cdots z_{n+m}}}\right) .
$$

Thus, if we denote $K=\sqrt[m]{z_{n+1} \cdots z_{n+m}}$, then the last column of $E(N)$ is $\left[\begin{array}{llll}z_{1} / K & \cdots & z_{n} / K & 1\end{array}\right]^{T}$. The proof of the third item is finished.

Note that the clustered matrix, $N$, and its priority vector are obtained by using the formulas in (i) and (iii), respectively. Both formulas are really straightforward since they involve exclusively simple (linear) vector operations to build the last column (row) of $N$, and replacing the last $m$ components of the priority vector by the $m$-th root of all of them.

According to this theorem there are no limitations regarding the size, $n+m$, of the matrix, nor with respect to the number, $m$, of items to be collapsed. Moreover, if the initial PCM has acceptable consistency, then this theorem guarantees consistency for the clustered structure; while if the initial PCM do not exhibit acceptable consistency, then it would be absurd to use this theorem to derive a clustered structure and claim consistency. Specifically, if $A \in \mathscr{M}_{n+m}$ is a consistent matrix, then item (ii) of Theorem 4 implies that, by collapsing the last $m$ judgements according to Definition 1, thus obtaining a skew-symmetric matrix $N \in \mathscr{M}_{n+1}$, matrix $E(N)$ is consistent. In other words, consistency is preserved by collapsing judgements. Furthermore, since all the involved operations are continuous, if matrix $A$ is close to being consistent (e.g., its consistency is acceptable according to Saaty's criterion), then one can apply Theorem 4 (approximately) to obtain the collapsed matrix and its priority vector. 


\section{Case study}

Despite worldwide efforts to improve water supply systems in recent years, intermittent water supply systems (IWSSs) still remain a harsh reality for water supply in many countries. An estimated one third of Africa and Latin America, and more than half of the Asian population have this type of supply [30].

By definition, IWSSs are those water services in which the supply is made for a limited amount of time. The usual frequency with which a new supply occurs is one day; however, in extreme cases, it may be more. In many small towns with an IWSS, service is for a number of hours for the entire network simultaneously; in larger cities, supply occurs in turns of several hours, the rotation being by service sectors.

Many reasons may be used to justify the existence of IWSSs. Many authors suggest three types of problems that can cause or perpetuate intermittent supply: poor technical management, economic scarcity, and absolute water scarcity [31, 32]. Increasing water scarcity due to climate change, and the demand intensification derived from the growing population may make the use of intermittent supply a too frequent practice [33].

Economic scarcity in developing countries -but with thriving water companies- increases the difficulties in achieving improvements in IWSSs, while, at the same time, only very basic operational information is available (pressure at some points, level of reservoirs and tanks, or measurement of just a few flow rates). Intermittent water supply operation and management in developing countries is mainly based on the experience of water company personnel, mainly derived from manual (in contrast to automatic) operation, and using simple offer-demand analyses [23]. In addition, the collection of quantitative information is deficient. So, by using a sector-operation-difficulty-related qualitative criterion [24] this limitation can be overcome. In addition, this criterion adds the experience of water company technicians into the decisionmaking process to improve the water system.

One way to minimize the difficulties resulting from IWSs is to enhance an adequate system of technical management. In this case study, the use of qualitative data, regarding the network sectors in which the system is divided, is analyzed as an interesting alternative for improving planning, operation, and maintenance in IWSSs.

The studied IWSS (see Figure 1) is one of the subsystems of the WDS of the city of Oruro (Bolivia). This subsystem is located in the southern part of the city, consists of 15 sectors fed by a single tank, and supplies water to 37,700 inhabitants. Each sector has a specific supply schedule and specific operation tasks, such as valve maneuvering, which are manually performed.

As said, one of the qualitative criteria of interest in an IWSS, and the subject of this study, is the difficulty of operation of each of the sectors. This variable depends on several factors such as the availability of sectorization valves, the certainty of their existence, whether existing valves are operating, whether they are visible or buried, the difficulty in working for operators, consumer complaints, and others. Being a complex qualitative criterion, technicians and water company experts who understand the operation of the network and its sectors were consulted. This also ensures active management in the process of improvement. The AHP multi-criteria analysis technique is used to rank the alternatives (obviously, the alternatives are the 15 sectors in which the network is currently sectorized), with respect to the single criterion 'difficulty of operation'.

AHP is a well established methodology for multi-criteria analysis that has been successfully applied in many fields such as environmental planning $[34,35]$, transport solutions $[36,37,38$, 


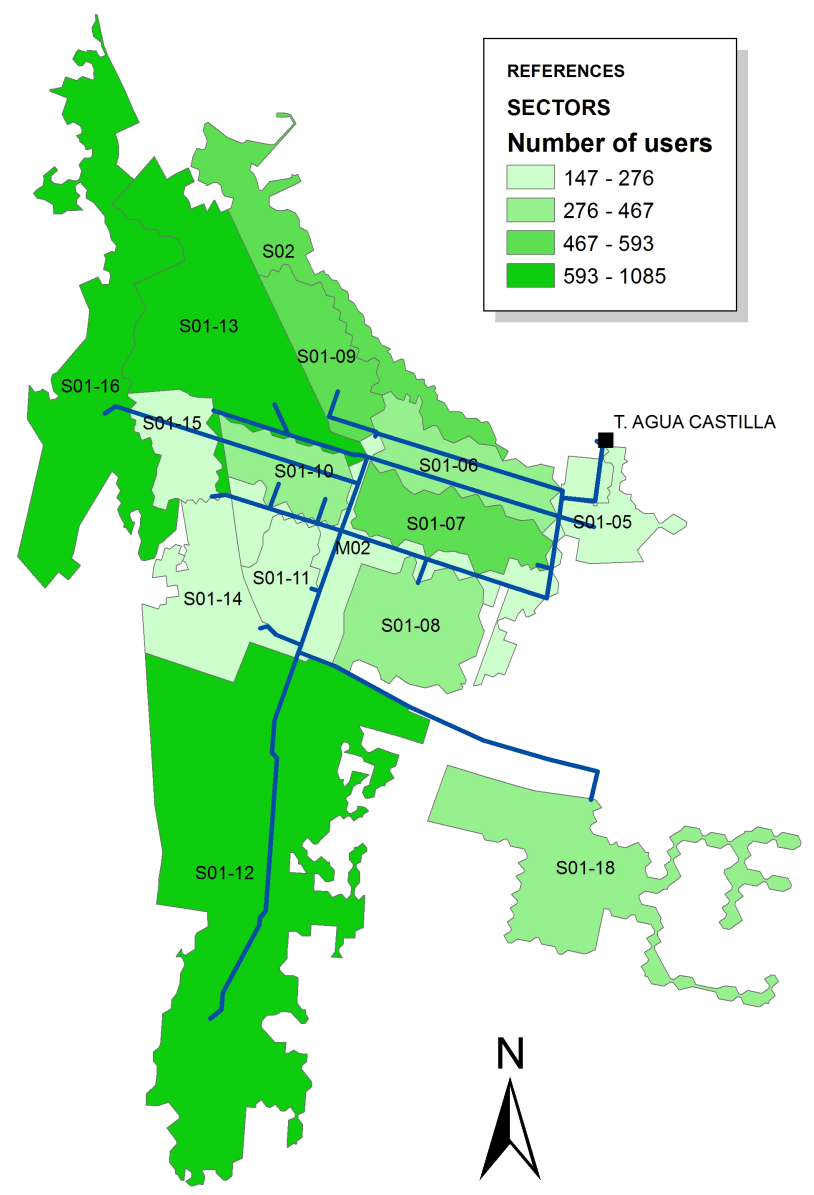

Figure 1: Studied IWSS, south area of Oruro, Bolivia.

39], social and educational sciences [40, 41], agriculture [42], to quote just a few fields with a few references. The success of this method is a result of its simplicity and robustness [43].

We proceeded with the pairwise comparison process, asking the experts to analyze the ease of operation of a sector with respect to another. Despite the large number of elements for comparison, the panel was able to develop a coherent and reliable comparison matrix (see Table 1). 


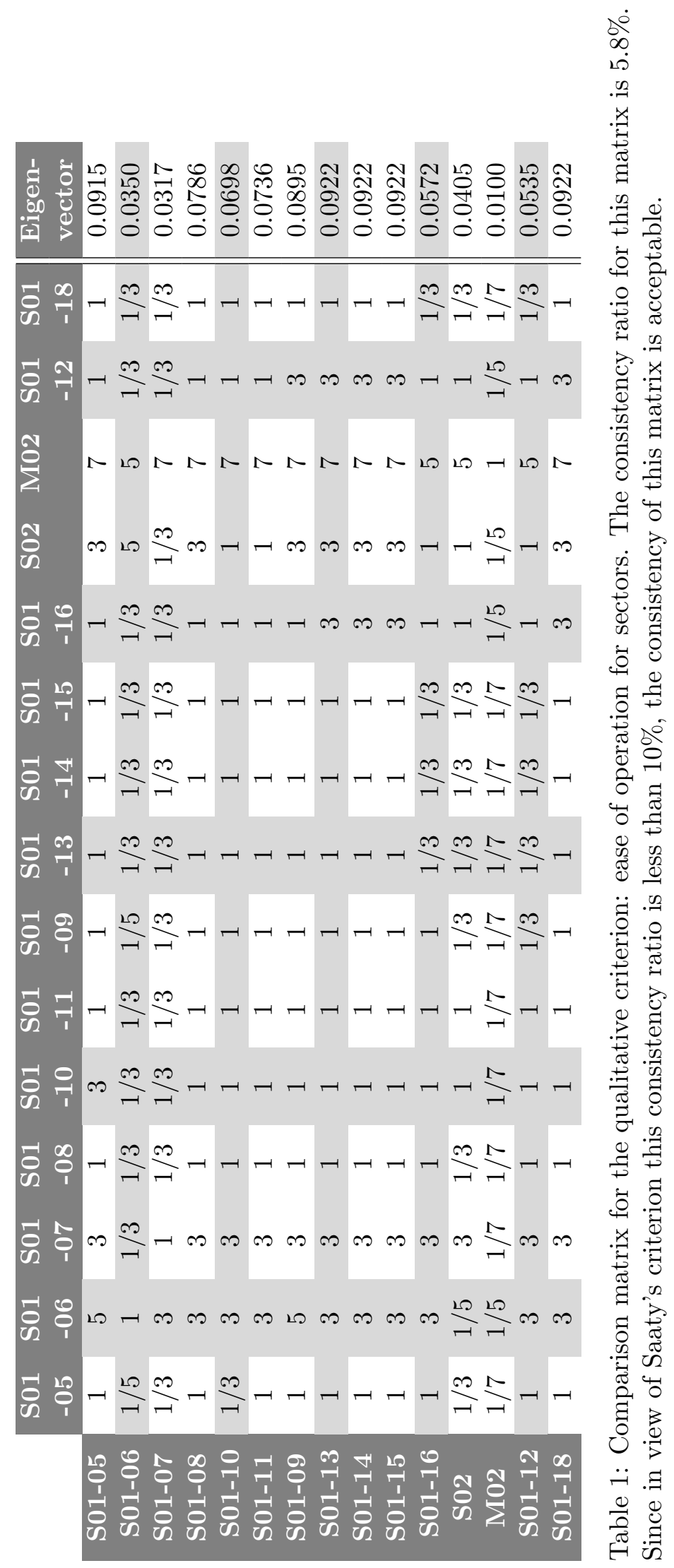


As explained before, the possibility of reducing the volume of this information may be deemed interesting, for example if posterior comparisons regarding alternatives were necessary. In addition, some patterns presented by the matrix clearly suggest the possibility of reducing the matrix size by grouping sectors, a situation that was not initially obvious.

The identification of these groups will enable us to develop strategies for improvement in the technical management based on differentiated areas.

Various proposals were presented to the technicians who manage the system. We highlight here two of them.

In the first proposal, guided solely by the number and distribution of values equal to 1 in the matrix, a consistent simplification was attempted with eight sectors grouped under a single item.

Specifically, sectors S01-18, S01-08, S01-10, S01-11, S01-09, S01-13, S01-14 and S01-15 were grouped, since after the permutation given by (S01-05, S01-06, S01-07, S01-16, S02, M02, S01-12, S01-18, S01-08, S01-10, S01-11, S01-09, S01-13, S01-14, S01-15), the entries of the $8 \times 8$ 'southeast' submatrix were all equal to one. The use of Theorem 4 for this situation provided the following $8 \times 8$ comparison matrix:

$\left[\begin{array}{cccccccc}1 & 5 & 3 & 1 & 3 & 7 & 1 & 1.082 \\ 0.2 & 1 & 0.333 & 0.333 & 5 & 5 & 0.333 & 0.414 \\ 0.333 & 3 & 1 & 0.333 & 0.333 & 7 & 0.333 & 0.375 \\ 1 & 3 & 3 & 1 & 1 & 5 & 1 & 0.676 \\ 0.333 & 0.2 & 3 & 1 & 1 & 5 & 1 & 0.479 \\ 0.143 & 0.2 & 0.143 & 0.2 & 0.2 & 1 & 0.2 & 0.118 \\ 1 & 3 & 3 & 1 & 1 & 5 & 1 & 0.633 \\ 0.924 & 2.414 & 2.666 & 1.479 & 2.09 & 8.463 & 1.58 & 1\end{array}\right]$.

The priority vector given by Theorem 4 is:

$$
\left[\begin{array}{llllllll}
0.227 & 0.087 & 0.079 & 0.142 & 0.100 & 0.025 & 0.132 & 0.209
\end{array}\right]^{T} .
$$

For this matrix, the Perron vector, corresponding to the Perron eigenvalue $\lambda=9.28$, is:

$$
\left[\begin{array}{llllllll}
0.253 & 0.126 & 0.106 & 0.184 & 0.124 & 0.024 & 0.183 & 0.223
\end{array}\right]^{T} .
$$

giving the values $C I=0.1831$ and $C R=13.08 \%$ for consistency index and consistency ratio, respectively. This last value exceeds the maximum allowed of $10 \%$.

Moreover, the obtained grouping was not satisfactory for technicians, since it grouped areas with clear different hydraulic behaviors.

The second proposal tried to use ideas provided by the technicians to guide the observation of patterns in the matrix of comparisons. Thus, sectors S01-09, S01-13, S01-14 and S01-15 were initially selected to be grouped. Theorem 4 above, by using the permutation given by (S01-05, S01-06, S01-07, S01-08, S01-10, S01-11, S01-16, S02, M02, S01-12, S01-18, S0109, S01-13, S01-14, S01-15), gives a new comparison matrix where the last four sectors are grouped (under the identification 09-15). The matrix, which corresponds to the sector order (S01-05, S01-06, S01-07, S01-08, S01-10, S01-11, S01-16, S02, M02, S01-12, S01-18, 09-15), is 
given by:

$$
\left[\begin{array}{cccccccccccc}
1 & 5 & 3 & 1 & 3 & 1 & 1 & 3 & 7 & 1 & 1 & 1 \\
0.2 & 1 & 0.333 & 0.333 & 0.333 & 0.333 & 0.333 & 5 & 5 & 0.333 & 0.333 & 0.383 \\
0.333 & 3 & 1 & 0.333 & 0.333 & 0.333 & 0.333 & 0.333 & 7 & 0.333 & 0.333 & 0.347 \\
1 & 3 & 3 & 1 & 1 & 1 & 1 & 3 & 7 & 1 & 1 & 0.858 \\
0.333 & 3 & 3 & 1 & 1 & 1 & 1 & 1 & 7 & 1 & 1 & 0.763 \\
1 & 3 & 3 & 1 & 1 & 1 & 1 & 1 & 7 & 1 & 1 & 0.804 \\
1 & 3 & 3 & 1 & 1 & 1 & 1 & 1 & 5 & 1 & 0.333 & 0.625 \\
0.333 & 0.2 & 3 & 0.333 & 1 & 1 & 1 & 1 & 5 & 1 & 0.333 & 0.442 \\
0.143 & 0.2 & 0.143 & 0.143 & 0.143 & 0.143 & 0.2 & 0.2 & 1 & 0.2 & 0.143 & 0.109 \\
1 & 3 & 3 & 1 & 1 & 1 & 1 & 1 & 5 & 1 & 0.333 & 0.585 \\
1 & 3 & 3 & 1 & 1 & 1 & 3 & 3 & 7 & 3 & 1 & 1.008 \\
1 & 2.614 & 2.885 & 1.165 & 1.311 & 1.244 & 1.601 & 2.262 & 9.161 & 1.711 & 0.993 & 1
\end{array}\right] .
$$

The priority vector given by Theorem 4 is:

$$
\left[\begin{array}{llllllllllll}
0.126 & 0.048 & 0.044 & 0.108 & 0.096 & 0.101 & 0.079 & 0.056 & 0.014 & 0.074 & 0.127 & 0.126
\end{array}\right]^{T} \text {. }
$$

For this matrix, the Perron vector, corresponding to the Perron eigenvalue $\lambda=13.2$, is:

$$
\left[\begin{array}{llllllllllll}
0.127 & 0.053 & 0.045 & 0.104 & 0.088 & 0.095 & 0.085 & 0.060 & 0.013 & 0.084 & 0.131 & 0.116
\end{array}\right]^{T} \text {. }
$$

giving the values $C I=0.1069$ and $C R=7.03 \%$, which is satisfactory from the consistency point of view. Continuing in the same line, a new clustering was performed, taking the latter as a starting point. In this case, sectors S01-08, S01-10 and S01-11 were the candidates for a new grouping.

Again, using Theorem 4 on the permutation of the previous matrix given by (S01-05, S01-06, S01-07, S01-16, S02, M02, S01-12, S01-18, 09-15, S01-08, S01-10, S01-11), a new comparison matrix was obtained with the last three sectors grouped (under the name 08-11), given by:

$$
\left[\begin{array}{cccccccccc}
1 & 5 & 3 & 1 & 3 & 7 & 1 & 1 & 1 & 1.238 \\
0.2 & 1 & 0.333 & 0.333 & 5 & 5 & 0.333 & 0.333 & 0.383 & 0.474 \\
0.333 & 3 & 1 & 0.333 & 0.333 & 7 & 0.333 & 0.333 & 0.347 & 0.429 \\
1 & 3 & 3 & 1 & 1 & 5 & 1 & 0.333 & 0.625 & 0.774 \\
0.333 & 0.2 & 3 & 1 & 1 & 5 & 1 & 0.333 & 0.442 & 0.548 \\
0.143 & 0.2 & 0.143 & 0.2 & 0.2 & 1 & 0.2 & 0.143 & 0.109 & 0.135 \\
1 & 3 & 3 & 1 & 1 & 5 & 1 & 0.333 & 0.585 & 0.724 \\
1 & 3 & 3 & 3 & 3 & 7 & 3 & 1 & 1.008 & 1.284 \\
1 & 2.614 & 2.885 & 1.601 & 2.262 & 9.161 & 1.711 & 0.993 & 1 & 1.239 \\
0.808 & 2.11 & 2.33 & 1.292 & 1.826 & 7.397 & 1.381 & 0.801 & 0.807 & 1
\end{array}\right] .
$$

In this matrix the corresponding order is (S01-05, S01-06, S01-07, S01-16, S02, M02, S01-12, S01-18, 09-15, 8-11).

The priority vector given by theorem 4 is:

$$
\left[\begin{array}{llllllllll}
0.159 & 0.061 & 0.055 & 0.099 & 0.070 & 0.017 & 0.093 & 0.160 & 0.159 & 0.128
\end{array}\right]^{T} \text {. }
$$


For this matrix, the Perron vector, corresponding to the Perron eigenvalue $\lambda=11.1$, is:

$$
\left[\begin{array}{llllllllll}
0.149 & 0.070 & 0.061 & 0.101 & 0.070 & 0.015 & 0.100 & 0.173 & 0.144 & 0.116
\end{array}\right]^{T} \text {. }
$$

giving the values $C I=0.125$ and $C R=8.40 \%$, which is still satisfactory from the consistency point of view.

This proposal was positively considered by the technicians. In addition to the acceptable values of $C R$, the technicians appreciated that both clusters, the initial 09-15 and the subsequent $08-11$, have an interesting technical interpretation, which is based on the proximity to the source of clusters 08-11 and 09-15 (which are at successive rings, consecutively further away from the source).

As a result, areas or groups of sectors with similar operating characteristics are defined based on the opinion of water company technicians. This poses a new scenario (see Figure 2) that will enable a better planning in the operation and maintenance tasks of the system, such as reorganizing the manual work of the operators, who hourly open and close the valves to supply water, and the prioritization of maintenance tasks. Therefore, it becomes a very useful tool for the technical management of intermittent water supply systems, and is a fundamental criterion for a future transition to a continuous water supply [22, 24, 23].

Regarding the procedure for obtaining the clusters of the considered elements, the authors think that details depend on the problem in hand. We think there are no general guidelines for this clustering process. In the two examples we have provided, completely different procedures are followed. In the case of the water supply system, it has been a trial and error procedure in close contact with the system experts. Several attempts were made, since there was no clear idea about which sectors to group and which not to group (except for some visual patterns identified in the original matrix). It was the final decision of the experts, after checking the various results produced, that was consolidated. As another example, if the technique had to be used to merge companies in shared markets to avoid the whole process of starting PCs from scratch once an alliance has been produced, the decision would consider companies inside and outside of the alliance. Conditions would probably be clear from the beginning, provided that the alliance process among companies is clear-cut. We believe that, in general, an a priori cluster structure with the best number of clusters may not exist and, consequently, we do not venture to state rules to this end. It would be a very interesting line of research for future study.

\section{Conclusions}

We have considered large AHP comparison matrices in this article. These matrices have scarcely been considered in the literature. One reason is related to the so-called 'channel capacity', a measure of our ability to process information that is based on the so-called Miller's magic number seven. However, inspired by other works on the basis of this magic number, we have reasoned that, in some cases, comparison matrices of a larger size may contain reliable information. Such is the case of the matrix presented in the case study. Another reason derives from the fact that recent information collected from databases and the internet has started to be handled through PCs. However, we are aware that the use of such large matrices may pose further difficulties. Therefore, we have designed a mechanism for reducing the size of a large comparison matrix by consistently compressing it, and eventually grouping some of the

original elements into clusters. To the best of the authors' knowledge there are no other similar 


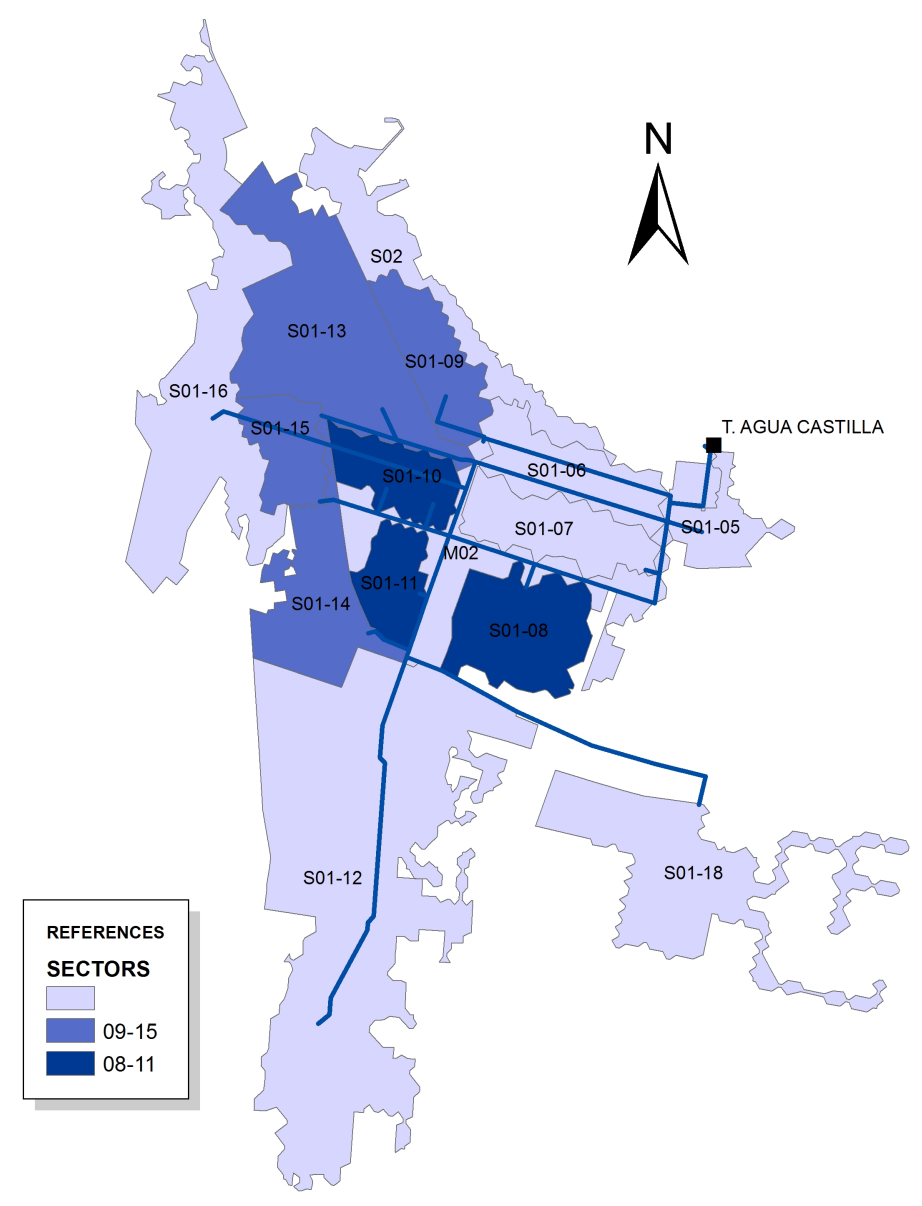

Figure 2: Studied IWSS, after cluster identification.

(a posteriori) compressing mechanisms for PCMs in the literature. In the case of judgment PCs, of course, such a reduction must be performed so that consistency is preserved and, in any case the experts must eventually validate any manipulation of their original comparison matrix. In general, this size reduction may be useful for various straightforward reasons, as explained in the introduction, derived from the obtained size reduction of the problem. However, in the case study addressed in this paper, we have additionally observed that the given mechanism has helped reveal non-explicit characteristics of the problem considered. Specifically, the identification of areas or groups of sectors with similar characteristics (pipe material, pipe age, etc.), based on the qualitative criteria provided by this technique has proven to be a very useful tool for improving the technical management of water systems. This is especially important for those systems with economic scarcity or mismanagement, where quantitative information is limited, if existing.

Although our case-study analysis is about IWSS, the tool we have developed may also be useful to obtain clusters of sectors in water systems with continuous supply, based on suitable 
qualitative characteristics of interest. This may be a future line of research within the water field.

From a wider perspective, the technique we have presented may also be useful in other decision-making contexts with shortages or a lack of quantitative information, and with many number of elements to be pairwise compared, and for which there is no clear a priori clustering. The benefits come from the possibility of compressing a comparison matrix consistently, and eventually clustering some of its entries, thus avoiding the need to perform a new PC process from scratch (as in the mentioned example regarding shared markets).

Finally, as explained above, we think there are no general guidelines for this clustering process. An a priori cluster structure with the best number of clusters may not exist. As a result, a study of adequate rules to this end is an appealing research subject for future study.

\section{Acknowledgment}

The use of English has been revised by John Rawlins, an associate member of the Institute of Translation and Interpreting (no. 9743) of the UK, 'Certified PRO' from ProZ according to the EN 15038.

\section{References}

[1] S. Bozóki, L. Csató and J. Temesi, An application of incomplete pairwise comparison matrices for ranking top tennis players, European Journal of Operation Research, 248(2016) 211-218, doi:10.1016/j.ejor.2015.06.069.

[2] W. Gu, M. Basu, Z. Chao and L. Wei, A unified framework for credit evaluation for internet finance companies: Multi-Criteria analysis through AHP and DEA, International Journal of Information Technology \& Decision Making 16(2017) 597-624, doi:10.1142/S0219622017500134.

[3] J.C. Leyva-Lopez, J.J. Solano-Noriega and D.A. Gastelum-Chavira, A Multi-Criteria approach to rank the municipalities of the states of Mexico by its marginalization level: The case of Jalisco, International Journal of Information Technology \& Decision Making 16(2017) 473-513, doi:10.1142/S0219622017500080.

[4] T.L. Saaty, A scaling method for priorities in hierarchical structures, Journal of Mathematical Psychology, 15(1977) 234-281, doi:10.1016/0022-2496(77)90033-5.

[5] Y. Shen, A. Hoerl and W. McConnell, An incomplete design in the Analytic Hierarchy Process, Mathematical and Computer Modelling, 16(1992) 121-129, doi:10.1016/08957177(92)90124-4.

[6] R. Islam, M. Biswal and S. Alam, Clusterization of alternatives in the Analytic Hierarchy Process, Military Operations Research, 3(1997) 69-78.

[7] E. Triantaphyllou, Linear programming based decomposition approach in evaluating priorities from pairwise comparisons and error analysis, Journal of Optimization Theory and Applications, 84 (1995) 207-234, doi:10.1007/BF02191743. 
[8] R. Islam and N.A. Abdullah, Management Decision-Making by the Analytic Hierarchy Process: A proposed modification for large-scale problems, Journal for International Business and Entrepreneurship Development, 3(2006) 18-40, doi:10.1504/JIBED.2006.011949.

[9] A. Ishizaka, A multicriteria approach with AHP and clusters for supplier selection, 15th International Annual Euroma Conference, Groningen (2008).

[10] A. Ishizaka, Clusters and pivots for evaluating a large number of alternatives in AHP, Brazilian Operations Research Society, 31(2012), doi:10.1590/S010174382012005000002 .

[11] G. Wright, Behavioral Decision Making. Plenum Press.

[12] E. Triantaphyllou, Multi-Criteria Decision Making Methods: A Comparative Study, (Vol. 44). Springer.

[13] E.R. Jalao, T. Wua and D. Shunk, An intelligent decomposition of pairwise comparison matrices for large-scale decisions, European Journal of Operational Research, 238(2014) 270-280, doi:10.1016/j.ejor.2014.03.032.

[14] M. Köksalan, V. Mousseau and S. Özpeynirci, Multi-Criteria sorting with category size restrictions, International Journal of Information Technology \& Decision Making, 16(2017) 5-23, doi:10.1142/S0219622016500061.

[15] S. Zhou, X. Xu, Y. Zhou and X. Chen, A large group decision-making method based of fuzzy preference relation, International Journal of Information Technology \& Decision Making, 16(2017) 881-897, doi:10.1142/S021962201550039X.

[16] T.L. Saaty and M.S. Ozdemir, Why the Magic Number Seven Plus or Minus Two, Mathematical and Computer Modelling, 38(2003) 233-244, doi:10.1016/S0895-7177(03)900835.

[17] M.S. Ozdemir, Validity and inconsistency in the analytic hierarchy process, Applied Mathematics and Computation, 161(2005) 707-720, doi:10.1016/j.amc.2003.12.099.

[18] G.A. Miller, The Magic Number Seven, Plus or Minus Two: Some Limits on Our Capacity for Processing Information, The Psychological Review, 63(1956) 81-97, doi:10.1037/h0043158.

[19] https://rapidbi.com/millermagicalnumberseven7/ (accessed on 15/10/2016)

[20] G. Marnell, Chunking the Information Presented to Readers. A critique of Information Mapping, The Australian and New Zealand Journal of Technical Communication, 33(2014), http://www.abelard.com.au/information\%20mapping.pdf.

[21] A. Baddeley, Working Memory, Thought, and Action, Oxford University Press, Oxford (2007).

[22] A.E. Ilaya-Ayza, Propuesta para la transición de un sistema con suministro de agua intermitente a suministro continuo. Doctoral Dissertation (2016) Universitat Politècnica de València. Valencia, Spain. 
[23] A.E. Ilaya-Ayza, E. Campbell, R. Pérez-Garca and J. Izquierdo, Network Capacity Assessment and Increase in Systems with Intermittent Water Supply, WATER, 8(4), 126 (2016), doi:10.3390/w8040126.

[24] A-E. Ilaya-Ayza, J. Benítez, J. Izquierdo and R. Pérez-Garca, Multi-criteria optimization of supply schedules in intermittent water supply systems, Journal of Computational and Applied Mathematics, 309(2017) 695-703, doi:10.1016/j.cam.2016.05.009.

[25] A. Certa, M. Enea, G. Galante and C. M. La Fata, Multi-objective human resources allocation in R\&D projects planning, International Journal of Production Research, 47(2009) 3503-3523, doi:10.1080/00207540701824233.

[26] K.H. Hu, W. Jianguo and G.H. Tzeng, Risk factor assessment improvement for China's cloud computing auditing using a new hybrid MADM model. International Journal of Information Technology \& Decision Making 16(2017) 737-777, doi:10.1142/S021962201750016X.

[27] J. Benítez, X. Delgado-Galván, J. Izquierdo and R. Pérez-García, Achieving matrix consistency in AHP through linearization, Applied Mathematical Modelling, 35(2011) 4449-4457, doi:10.1016/j.apm.2011.03.013.

[28] J. Benítez, J. Izquierdo, R. Pérez-García and E. Ramos-Martínez, A simple formula to find the closest consistent matrix to a reciprocal matrix, Applied Mathematical Modelling, 38(2014) 3968-3974, doi:10.1016/j.apm.2014.01.007

[29] T. Saaty, Relative Measurement and its Generalization in Decision Making: Why Pairwise Comparisons are Central in Mathematics for the Measurement of Intangible FactorsThe Analytic Hierarchy/Network Process, Royal Spanish Academy of Sciences, Series A, Mathematics, 102 (2)(2008) 251-318, http://www.rac.es/ficheros/doc/00576.PDF

[30] C. Van den Berg and A. Danilenko, The IBNET Water Supply and Sanitation Performance Blue Book. Washington D.C.: The World Bank, 2011.

[31] N. Totsuka, N. Trifunovic and K. Vairavamoorthy, Intermittent urban water supply under water starving situations, 30th WEDC International Conference, 505-512. Vientiane, Lao (2004).

[32] B. Charalambous, The Effects of Intermittent Supply on Water Distribution Networks. Water Loss 2012, Manila, Philippines (2012).

[33] S. Tsegaye, J. Eckart and K. Vairavamoorthy, Urban water management in cities of the future: emerging areas in developing countries, On the water front (2011) 42-48.

[34] X. Delgado-Galván, J. Izquierdo, J. Benítez and R. Pérez-Garca, Joint Stakeholder Decision-Making on the Management of the Silao-Romita Aquifer Using AHP, Environmental Modelling \& Software, 51(2014) 310-322, doi:10.1016/j.envsoft.2013.10.008.

[35] M. Bottero, E. Comino and V. Riggio, Application of the Analytic Hierarchy. Process and the Analytic Network Process for the assessment of different wastewater treatment systems, Environmental Modelling \& Software, 26(2010) 1211-1224, doi:10.1016/j.envsoft.2011.04.002. 
[36] A. Awasthi and S.S. Chauhan, Using AHP and Dempstere-Shafer theory for evaluating sustainable transport solutions, Environmental Modelling \& Software, 26 (2011) 787-796, doi:10.1016/j.envsoft.2010.11.010.

[37] T. Lupo, Handeling stakeholder uncertain judgments in strategic transport service analyses, Transport policy, 29(2013) 54-63, doi:org/10.1016/j.tranpol.2013.04.002.

[38] M. Berrittella, A. Certa, M. Enea and P. Zito, Transport policy and climate change: how to decide when experts disagree?, Environmental Science \& Policy, 11(2008) 307314, doi:10.1016/j.envsci.2008.01.008.

[39] M.M. Kablan, Decision support for energy conservation promotion: an analytic hierarchy process approach, Energy Policy 32(2004) 1151-1158, doi:10.1016/S0301-4215(03)000788.

[40] A. Certa A, M. Enea, G. Galante and M. La Fata Concetta, Multi-objective Human Resources Allocation in R\&D Projects Planning, International Journal of Production Research, 47(2009) 3503-3523, doi:10.1080/00207540701824233.

[41] A. Certa, M. Enea and Hopps, A multi-criteria approach for the group assessment of an academic course: a case study, Studies in Educational Evaluation 44(2015) 16-22, doi:10.1016/j.stueduc.2014.12.001.

[42] L. Gao and A. Hailu, Ranking management strategies with complex outcomes: an AHP-fuzzy evaluation of recreational fishing using an integrated agent-based model of a coral reef ecosystem, Environmental Modelling \& Software 31(2012) 3-18, doi:10.1016/j.envsoft.2011.12.002.

[43] L.G Vargas, An overview of the analytic hierarchy process and its applications, European Journal of Operational Research 48(1990) 2-8, doi:10.1016/0377-2217(90)90056-H. 